\title{
Analogs for Unlayered-Graphene Droplet-Formation in Stellar Atmospheres.
}

\author{
P. Fraundorf ${ }^{1,3}$, Melanie Lipp ${ }^{2,1}$ and Taylor Savage ${ }^{1}$. \\ 1. Physics \& Astronomy \& Center for Nanoscience, University of Missouri - St Louis, MO, 63121, USA \\ 2. Universität Stuttgart, 70550 Stuttgart, Germany, EU. \\ 3. Physics Department, Washington University - St Louis, Mo, 63110, USA
}

Micron-sized presolar carbon-spheres condensed before the origin of our solar system, in the atmosphere of asymptotic-giant-branch stars from carbon atoms dredged up following nucleosynthesis in the stellar interior, have been extracted by dissolution from primitive carbonaceous chondrite meteorites, identified by noble-gas and major-element isotopic analysis, and available to electron microscopists on earth for over two decades [1-4]. Some of these spheres appear to consist of graphite condensed on carbide grains, but others instead show "graphite-onion" rims that surround a spherical-core of unlayeredgraphene i.e. carbon that in diffraction shows graphite (hk0) spacings only. The high spatial-frequency tail of these graphene lines [5] suggests a graphene-sheet coherence-width in the 4-nm size range [1], consistent with electron-phase-contrast images of single (sometimes segmented) edge-on sheets $[2,4]$.

Three key questions are: What process in a low-pressure atmosphere can form condensed spheres of graphene with densities approaching that of graphite, but with graphitic-layering absent? How might we synthesize such material in the laboratory? What interesting properties might it have as a result?

Our group has been focusing on three aspects of this problem: (i) Measurements of core-material density (relative to the graphite rim) in ultra-microtomed spheres using zero-loss/deflection analysis [6] via energy-filtered transmission electron microscopy or TEM, (ii) modeling of graphene-sheet nucleation and growth from a carbon melt to get atom-positions for TEM image simulation and estimate the likelihood of pentagonal-loop nucleation as explanation of apparent edge-on facets [4] using the Tersoff potential [7], and (iii) the laboratory synthesis of unlayered-graphene via slow-cooling of carbon vapor.

Carbon plasmon-peak energies [1] suggest an unlayered graphene-density near the 2.3[g/cc] of graphitic carbon. Zero-loss/deflection analysis [6] shows promise for inelastic mean-free-path mapping in a wide range of disordered materials, but specular reflection by diffraction can complicate life (e.g. via Pendullosung or thickness-fringe contrast) by putting electrons into "Bragg storage" if the crystals are thick enough for multiple scattering within the same crystal. Although our first analyses suggest a density closer to $1.8[\mathrm{~g} / \mathrm{cc}]$ for presolar unlayered-graphene, this may not be confirmed without further calibration studies on the presolar specimens themselves.

Condensation of particles in a low-pressure stellar-atmosphere will require local density increases [1] e.g. associated with jets of material around sunspots, and might take place one $\mathrm{C}$ atom at a time, by clustering of polycyclic aromatic hydrocarbons, or by condensation of droplets from a carbon vapor. The latter has the advantage that it will give us the spherical core-shape and density. Although thermodynamics predicts that below pressures of $10.8[\mathrm{MPa}]$, carbon on warming sublimates to a vapor at around $3915 \mathrm{~K}$, containerless metals on cooling generally supercool in the liquid state until as much as $30 \%$ below the liquid condensation temperature [8], depending on the amount of rearrangement needed between liquid and solid state configurations. 
A liquid condensation temperature for carbon around $4600 \mathrm{~K}$, in this context, along with the major rearrangement between 12-neighbor coordination in an fcc liquid and the graphene-sheet structure, thus suggest that supercooling to temperatures as low as $3220 \mathrm{~K}$ (2950C) might be expected in the absence of condensation seeds (like the carbide grains seen at the center of other particles). This synthesis path might also explain the possible TEM evidence $[2,4]$ that the graphene sheets are flat, except where they are seen edge-on to connect at sharp bends whose geometry is suggestive of nucleation on pentagonal (rather than hexagonal) carbon-loops. So far, we are assembling graphene-sheet and graphene-pentacone structures for TEM image simulation (Fig. 1), but only beginning to look at simulated annealing models of pentagonal versus hexagonal loop nucleation.

Finally, slow-cooled carbon-vapor synthesis in the inside of a resistively-evaporating carbon-cylinder now looks possible. However we are only beginning to examine carbon spheres created as a result.

\section{References:}

[1] Thomas J. Bernatowicz et al. (1996), Astrophysical Journal 472, 760.

[2] P. Fraundorf and Martin Wackenhut (2002) Ap. J. Lett. 578(2) L153-156.

[3] Thomas K. Croat et al. (2005) Astrophysical Journal 631, 976-987.

[4] Eric Mandell (2007) Ph.D. Dissertation at UM-St Louis/Rolla.

[5] B. E. Warren (1941) "X-ray diffraction in random layer lattices", Phys. Rev. 59(9), 693.

[6] P. Fraundorf et al., (2010) Microscopy and MicroAnalysis 16:S2, 1534-1535 arXiv:1203.6342.

[7] J. Tersoff (1988) Phys. Rev. Lett. 61:25, 2879-2882.

[8] K. F. Kelton and A. L. Greer (2010) Nucleation in Condensed Matter (Elsevier, Amsterdam).

[9] Thanks to Roy Lewis at U. Chicago and T. J. Bernatowicz for the presolar specimens used here.

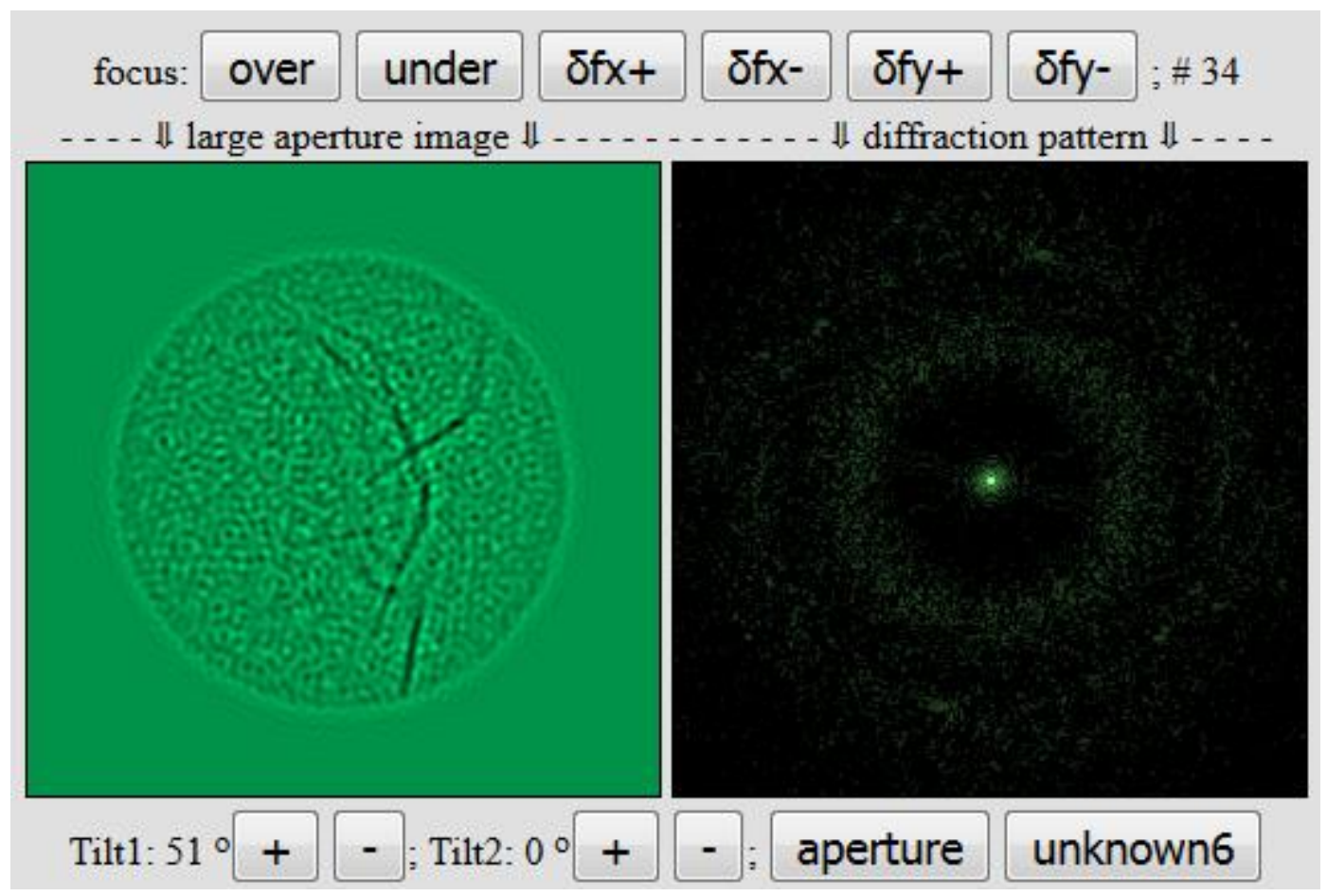

Figure 1. On-line strong-phase-object simulation of 15 faceted-nanocones in an 8-nm sphere with 26,846 "non-touching" C atoms. The projected-potential power-spectrum at right shows graphene $\{100\}$ and $\{110\}$ rings, as well as rel-rods associated with some of the edge-on graphene-sheets. 